\title{
Structure in the classical knot concordance group
}

\author{
Tim D. Cochran, Kent E. Orr and Peter Teichner*
}

\begin{abstract}
We provide new information about the structure of the abelian group of topological concordance classes of knots in $S^{3}$. One consequence is that there is a subgroup of infinite rank consisting entirely of knots with vanishing Casson-Gordon invariants but whose non-triviality is detected by von Neumann signatures.
\end{abstract}

Mathematics Subject Classification (2000). 57M25.

Keywords. Knot concordance, von Neumann signatures, Blanchfield pairing, Casson-Gordon invariants.

\section{Introduction}

Let $\mathcal{C}$ be the abelian group of topological concordance classes of knots in $S^{3}$. In this paper we provide new information about its structure. One consequence is that there is a subgroup of infinite rank consisting entirely of knots with vanishing Casson-Gordon invariants [CG] but whose non-triviality is detected by the von Neumann signature invariants of [COT].

Recall that two knotted circles in $S^{3}$ are topologically concordant if there is a locally flat topological embedding of the annulus in $S^{3} \times[0,1]$ whose restriction to the boundary components gives the knots. The equivalence class of the trivial knot is the identity for $\mathcal{C}$, which is a group under connected-sum of knots, and inverses are given by taking the mirror-image and reversing the string orientation. A slice knot is one which is zero in this group, or, equivalently bounds a locally-flat embedded disk in $B^{4}$. All of our work is in this topological category.

In $[\mathrm{COT}]$ we defined a geometric filtration of $\mathcal{C}$

$$
0 \subset \cdots \subset \mathcal{F}_{n .5} \subset \mathcal{F}_{n} \subset \cdots \subset \mathcal{F}_{1.5} \subset \mathcal{F}_{1} \subset \mathcal{F}_{.5} \subset \mathcal{F}_{0} \subset \mathcal{C}
$$

where $\mathcal{F}_{h}$ consists of all $h$-solvable knots for $h \in \frac{1}{2} \mathbb{N}_{0}$. An equivalent and more algebraic definition of these terms is reviewed in the next section. We showed that 0 -solvable knots are precisely the Arf invariant zero knots so that $\mathcal{C} / \mathcal{F}_{0} \cong \mathbb{Z}_{2}$ given by the Arf invariant and that $\mathcal{C} / \mathcal{F}_{.5}$ is J. P. Levine's algebraic concordance group 
which he proved in $[\mathrm{L}]$ was isomorphic to $\mathbb{Z}^{\infty} \oplus\left(\mathbb{Z}_{2}\right)^{\infty} \oplus\left(\mathbb{Z}_{4}\right)^{\infty}$. We also showed that knots in $\mathcal{F}_{1.5}$ have vanishing Casson-Gordon invariants and thus $\mathcal{F}_{1} / \mathcal{F}_{1.5}$ has infinite rank, as detected by Casson-Gordon invariants (the last statement goes back to Jiang $[J])$. Finally, we showed that $\mathcal{F}_{2} / \mathcal{F}_{2.5}$ is non-zero, in particular an infinite set of non-slice knots with vanishing Casson-Gordon invariants was exhibited. Here we will show that this set of classes is linearly independent.

More precisely,

Theorem. $\mathcal{F}_{2} / \mathcal{F}_{2.5}$ has infinite rank.

The infinite set $\left\{K_{i}\right\}$ of non-trivial elements of $\mathcal{F}_{2} / \mathcal{F}_{2.5}$ that was exhibited in [COT, Sec. 6] was obtained from a single "seedling" ribbon knot by certain satellite constructions (which we call "genetic modifications") using a sequence of auxiliary knots $\left\{J_{i}\right\}$. Non-triviality was proven by evaluating a von Neumann signature whose value on $K_{i}$ was shown to be essentially the integral of the classical Levine-Tristram signature function of $J_{i}$. This pleasing fact is verified in Section 5 of this paper. It is simple enough to find a set $\left\{J_{i}\right\}$ for which the set of these real numbers is integrally linearly independent (and we do this in Section 5 of the present paper). If our higher-order invariants were additive under connected-sum, then the Theorem would follow immediately. However, the higher-order nature of our invariants (just as for those of Casson and Gordon) makes it difficult even to formulate an additivity statement. In particular, our third-order invariants depend on choices of "metabolizers" (or self-annihilating submodules) for the $1^{\text {st }}$ and $2^{\text {nd }}$-order Blanchfield-Seifert forms, and unfortunately, the number of such choices is usually infinite for any connected sum of knots. To avoid the difficulties of the obvious direct approach, we employ a slight variation which makes crucial use of a special technical feature of the $K_{i}$ (arising from the corresponding fact for the original ribbon knot), namely that they have unique $1^{\text {st }}$ and $2^{\text {nd }}$-order metabolizers.

Only the seemingly technical problem of finding a "seedling" ribbon knot with unique metabolizers for its higher order linking forms (of orders $1,2, \ldots, n-1$ ) obstructs us from using the very same proof to show that $\mathcal{F}_{n} / \mathcal{F}_{n .5}$ has infinite rank for each $n \in \mathbb{Z}_{+}$.

Remark. In very recent work $[\mathrm{CT}]$ two of us have shown that $\mathcal{F}_{n} / \mathcal{F}_{n .5}$ has nonzero rank for each $n \geq 0$ but the proof does not seem to adapt to show infinite rank. The latter is still open for $n>2$.

We also include in Section 5 a proof of the following theorem about genus one slice knots (more generally about 1.5-solvable knots), that was announced in [COT]. It should be compared and contrasted to Theorem 4 of [Gi]. In the case of slice knots, this result first appeared as a corollary to Theorem 3.13 in the unpublished Ph.D. thesis of D. Cooper. 
Theorem. Suppose $K$ is a 1.5-solvable knot (for example a slice knot) whose Alexander polynomial is not 1 and which admits a Seifert surface $F$ of genus 1 . Then there exists a homologically essential simple closed curve, $J$, on $F$ that has self-linking number zero and such that the integral of the Levine-Tristram signature function of $J$ vanishes.

In the proof, we first identity the integral of the Levine-Tristram signatures of $J$ as the von Neumann signature, $\sigma_{\mathbb{Z}}^{(2)}(J)$, of $J$ with respect to the homomorphism onto $\mathbb{Z}$, see Proposition 5.1. Then we use the naturality of von Neumann's continuous dimension to identify $\sigma_{\mathbb{Z}}^{(2)}(J)$ with the von Neumann signature, $\sigma_{\Gamma}^{(2)}(K)$, of the original knot $K$ with respect to a homomorphism to a certain metabelian group $\Gamma$. Compared to the other proofs of this result, our argument gives a more direct reason why this real number is a concordance invariant of $K$.

We wish to thank Andrew Ranicki and Michael Larsen for helpful contributions.

\section{2. $n$-solvable knots and von Neumann $\rho$-invariants}

We briefly review some of the definitions of [COT] which are used herein.

Let $G^{(i)}$ denote the $i$-th derived group of a group $G$, inductively defined by $G^{(0)}:=G$ and $G^{(i+1)}:=\left[G^{(i)}, G^{(i)}\right]$. A group is $n$-solvable if $G^{(n+1)}=1$. For a CW-complex $W$, we define $W^{(n)}$ to be the regular covering space corresponding to the subgroup $\pi_{1}(W)^{(n)}$. If $W$ is an spin 4-manifold then there is an intersection form

$$
\lambda_{n}: H_{2}\left(W^{(n)}\right) \times H_{2}\left(W^{(n)}\right) \longrightarrow \mathbb{Z}\left[\pi_{1}(W) / \pi_{1}(W)^{(n)}\right]
$$

and a self-intersection form $\mu_{n}$ (see [Wa] chapter 5 and [COT] section 7). An $n$-Lagrangian is a $\mathbb{Z}\left[\pi_{1}(W) / \pi_{1}(W)^{(n)}\right]$-submodule $L \subset H_{2}\left(W^{(n)}\right)$ on which $\lambda_{n}$ and $\mu_{n}$ vanish and which maps (under the covering map) onto a (1/2)-rank direct summand of $H_{2}(W ; \mathbb{Z})$. An $n$-surface $F$ in $W$ is a based, immersed surface in $W$, which lifts to $W^{(n)}$. Thus $\lambda_{n}$ and $\mu_{n}$ can be computed in $W$ by considering intersections weighted by elements of $\pi_{1}(W) / \pi_{1}(W)^{(n)}$, and an $n$-Lagrangian may be conveniently encoded by considering a collection of $n$-surfaces whose lifts generate it.

Suppose $K$ is a knot and $M$ is the closed 3-manifold resulting from 0 -framed surgery on $S^{3}$ along $K$.

Definition 2.1. A knot (or $M$ ) is n-solvable $\left(n \in \mathbb{N}_{0}\right)$ if $M$ bounds a spin 4manifold $W$, such that the inclusion map induces an isomorphism on first homology and such that $W$ admits two dual $n$-Lagrangians. This means that $\lambda_{n}$ pairs the two Lagrangians non-singularly and that their images together freely generate $H_{2}(W)$. Such a $W$ is called an $n$-solution for $K$ (or for $M$ ). Note that the exterior of a slice disk is, for any $n$, an $n$-solution for the slice knot (and for $M$ ) simply because the second integral homology vanishes.

A knot is (n.5)-solvable, $n \in \mathbb{N}_{0}$, if, in addition to the above, one of the dual 
$n$-Lagrangians is the image (under the covering map) of an $(n+1)$-Lagrangian. Then $W$ is called an (n.5)-solution for $K$ (or for $M$ ).

The set $\mathcal{F}_{n}$ (respectively $\mathcal{F}_{(n .5)}$ ) of concordance classes of $n$-solvable (respectively (n.5)-solvable) knots is a subgroup of $\mathcal{C}$.

More details can be found in [COT] sections 1, 7 and 8 .

Suppose $\phi: \pi_{1}(M) \longrightarrow \Gamma$ is a homomorphism. The von Neumann $\rho$-invariant $\rho(M, \phi) \in \mathbb{R}$ is then defined and satisfies $\rho(-M, \phi)=-\rho(M, \phi)[\mathrm{ChG}$. Recall [COT, Definition 5.8 and Lemma 5.9] that whenever $(M, \phi)=\partial(W, \psi)$ for some compact, oriented 4-manifold $W, \rho(M, \phi)=\sigma_{\Gamma}^{(2)}(W, \psi)-\sigma_{0}(W)$ where $\sigma_{\Gamma}^{(2)}$ is the von Neumann signature of the intersection form on $H_{2}(W ; \mathbb{Z} \Gamma)$ and $\sigma_{0}$ is the ordinary signature. If $\Gamma$ is a poly-torsion-free-abelian group (henceforth PTFA) then $\mathbb{Z} \Gamma$ embeds in a skew field of fractions $\mathcal{K}_{\Gamma}$ and $\sigma_{\Gamma}^{(2)}$ may be viewed as a realvalued homomorphism from $L^{0}\left(\mathcal{K}_{\Gamma}\right)$, so $\sigma_{\Gamma}^{(2)}(W, \psi)$ is a function of the Witt class of the intersection form on the free module $H_{2}\left(W ; \mathcal{K}_{\Gamma}\right)$.

We shall need only the following properties of $\rho$ from [COT].

(2.2) If $\Gamma$ is an $n$-solvable PTFA group and $\phi$ extends over some (n.5)-solution $W$ for $M$, then $\rho(M, \phi)=0$ [COT, Theorem 4.2]. In particular, if $K$ is a slice knot and $\phi$ extends over the exterior of a slice disk then $\rho(M, \phi)=0$ for any PTFA group $\Gamma$. The reader who is not familiar with $[\mathrm{COT}]$ can see that the latter follows from the very believable fact that $H_{2}(W ; \mathbb{Z})=0$ implies that $H_{2}$ of the $\Gamma$-cover of $W$ is $\Gamma$-torsion, hence $H_{2}\left(W ; \mathcal{K}_{\Gamma}\right)=0$ [COT, Prop. 4.3].

(2.3) (subgroup property) If $\phi$ factors through a subgroup $\Gamma^{\prime}$, then $\rho(M, \phi)=$ $\rho\left(M, \phi_{0}\right)$ where $\phi_{0}: \pi_{1}(M) \longrightarrow \Gamma^{\prime}$ is the induced factorization of $\phi[\mathrm{COT}$, Proposition 5.13]. This is a consequence of the corresponding fact for the canonical trace on a group von Neumann algebra.

(2.4) If $\Gamma=\mathbb{Z}$ and $\phi$ is non-trivial then $\rho(M, \phi)=\int_{z \in S^{1}} \sigma(h(z)) d z-\sigma_{0}(h)$ if $h$ is a matrix representing the intersection form on

$$
H_{2}\left(W ; \mathbb{C}\left[t, t^{-1}\right]\right) / \text { torsion }
$$

and $\sigma_{0}$ is the ordinary signature [COT, Lemma 5.4, Def. 5.3] (in fact we prove in the Appendix that $\rho(M, \phi)$ is the integral of the Levine-Tristram signature function of the $\operatorname{knot} K$ normalized to have value 0 at $z=1$, although this more precise fact is not strictly needed).

(2.5) If $\phi$ is the trivial homomorphism then $\rho(M, \phi)=0$.

If $K$ is an oriented knot then there is a canonical epimorphism $\phi: \pi_{1}(M) \longrightarrow \mathbb{Z}$. If $K$ has Arf invariant zero then there exists a spin 4-manifold $W$ and a map $\psi: \pi_{1}(W) \longrightarrow \mathbb{Z}$ such that $\partial(W, \psi)=(M, \phi)$. In fact we can assume $\pi_{1}(W) \cong \mathbb{Z}$. Such a $W$ is then a 0 -solution for $K$ (for $M$ ). In particular, $\rho(M, \phi)$ is always defined for such $\phi$. Let $\rho(K)$ denote this canonical real number (the integral of the normalized Levine-Tristram signature function of $K$ ). Note $M_{-J}=-M_{J}$ so $\rho(-J)=-\rho(J)$. The proof of the following technical result is deferred to Section 5 . 
Proposition 2.6. There exists an infinite set $\left\{J_{i} \mid i \in \mathbb{Z}_{+}\right\}$of Arf invariant zero knots such that $\left\{\rho\left(J_{i}\right)\right\}$ is linearly independent over the integers.

\section{Constructing $n$-solvable knots by genetic modification}

We describe a satellite construction, that we call "genetic modification", in which a given seed knot $K$ is altered by an infection knot $J$ along an axis $\eta$. We then evaluate the effect of such an alteration on the von Neumann $\rho$-invariants introduced in section 2. The construction is a special case of J. Levine's knot realization construction via surgery on links and can be summarized as follows.

Seize a collection of parallel strands of $K$ in one hand, just as you might grab some hair in preparation for braiding. Then, treating the collection as a single fat strand, tie it into the knot $J$. For example, applying this to a single strand of $K$ has the effect of altering $K$ by the addition of the local knot $J$. This would be a rather radical alteration. Applying this to two strands of $K$ which are part of a "band" of a Seifert surface for $K$ has the effect of tying that band into a knot $J$. In this simple case the construction agrees with the one used often by A. Casson, C. Gordon, P. Gilmer, R. Litherland, D. Ruberman, C. Livingston and others to create knots with identical Alexander modules but different Casson-Gordon invariants. Various other versions of this construction have been useful in knot and link theory (see e.g. [Lt], [Li], [CO], [C]).

In the application relevant to this paper, we will choose the seed knot $K$ to be a ribbon knot and choose a circle in $S^{3} \backslash K$ (the axis of the modification) which lies deep in the derived series of $\pi_{1}\left(S^{3} \backslash K\right)$ and yet bounds an embedded disk in $S^{3}$. The knot will pierce this disk many times. The alteration is to cut open $K$ along this disk and tie all the strands passing through into a knot $J$ which we call the infection (compare Definition 3.1 and the right-most part of Figure 1).

We named this procedure a "genetic modification" because, as opposed to a general surgery, its controlling parameter, the axis, involves very precise and subtle knowledge of the derived series of the knot group. A DNA strand is a million times longer than the diameter of a cell and topoisomerases miraculously find the precise locations on this strand to perform their genetic modifications. Analogously, the knot group is infinitely long as measured by the derived series [C, Corollary 4.8] and we use extremely precise control of this when choosing the axis (especially in $[\mathrm{CT}])$. On the other hand, the infection knots $J$ that we use in the modification are quite robust just like a virus seems to be a very robust thing.

This should be contrasted to the construction proposed by Casson-Gordon (to create candidates for non-slice knots with vanishing Casson-Gordon invariants) and to the more recent modification constructions of T. Stanford (inserting braids) and K. Habiro (simple clasper modification). There one uses very little control on the axis of the modification but the infecting knot $J$ is a subtle knot (e.g. has vanishing Casson-Gordon invariants or has vanishing finite type invariants up to 
a given degree).

Details of the general construction follow.

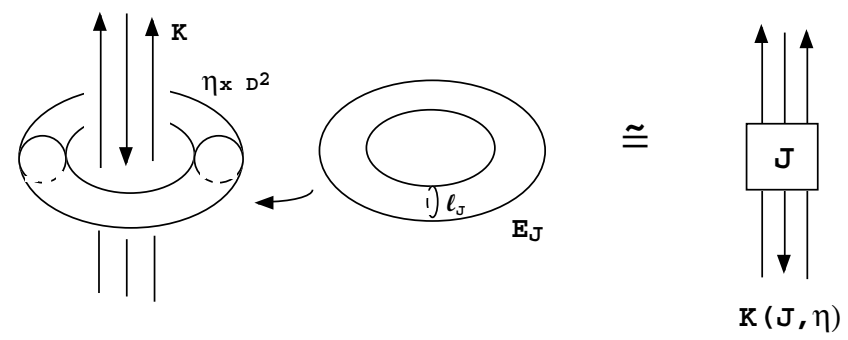

Figure 1

Let $M$ and $M_{J}$, respectively, denote the zero framed surgeries on the knots $K$ and $J$, and $E$ and $E_{J}$ denote their exteriors. Suppose $\eta$ is an oriented simple closed curve in $E$, which is unknotted in $S^{3}$. Choose an identification of a tubular neighborhood of $\eta$ with $\eta \times D^{2}$ in such a way that $\eta \times\{1\} \subset \eta \times \partial D^{2}$ is a longitude $\ell_{\eta}$, and $\{*\} \times \partial D^{2}$ is a meridian $\mu_{\eta}$. Form a new oriented manifold $E^{\prime}=\left(E-\operatorname{int}\left(\eta \times D^{2}\right)\right) \cup E_{J}$ by an identification of $\partial E_{J}$ with $\eta \times \partial D^{2}$ which sends $\mu_{\eta}^{-1}$ to the longitude of $J$, denoted $\ell_{J}$, and sends $\ell_{\eta}$ to $\mu_{J}$. Note that $\partial E^{\prime}=\partial E=$ $K \times S^{1}$ and since $E \cup\left(K \times D^{2}\right)=S^{3}, E^{\prime} \cup\left(K \times D^{2}\right)=\left(S^{3}-\operatorname{int}\left(\eta \times D^{2}\right)\right) \cup E_{J}$. Since $\eta$ is unknotted, $S^{3}-\operatorname{int}\left(\eta \times D^{2}\right)$ is a solid torus ST and $\mathrm{ST} \cup E_{J} \cong S^{3}$ as can be confirmed by checking the identifications. Hence $E^{\prime} \cup\left(K \times D^{2}\right) \cong S^{3}$. Therefore $E^{\prime}$ is the exterior $S^{3} \backslash K^{\prime}$ of a knot $K^{\prime}$ which is the image of $K$ under the identification $E^{\prime} \cup\left(K \times D^{2}\right) \cong S^{3}$.

Definition 3.1. The new knot $K^{\prime}=K(J, \eta)$ is called the genetic modification of the seed knot $K$ with the infection knot $J$ along the axis $\eta$. Since $K \subset \mathrm{ST}, K^{\prime}$ is a satellite of $J$.

It is left to the reader to see that $K^{\prime}$ is indeed the result of tying the strands of $K$ that "pass through the 2-disk spanned by $\eta$ " into the knot $J$. Finally, if we let $M^{\prime}$ denote the zero framed surgery on $K^{\prime}$, then $M^{\prime}=\left(M-\operatorname{int}\left(\eta \times D^{2}\right)\right) \cup E_{J}$. We shall show the following:

Proposition 3.1. If $K$ is n-solvable, $\eta \in \pi_{1}(M)^{(n)}$ and $J$ has Arf invariant zero, then $K^{\prime}=K(J, \eta)$ is n-solvable.

Hence one speculates that if one begins with a slice knot $K$, which is of course $n$-solvable for each $n$, and $\eta \in \pi_{1}(M)^{(n)}-\pi_{1}(M)^{(n+1)}$, then many different $n$ solvable knots could be constructed as long as the derived series of $\pi_{1}(M)$ does not stabilize. In fact it is proved in $[\mathrm{C}]$ that the derived series of a knot group cannot stabilize unless it has Alexander polynomial 1 and that of $\pi_{1}(M)$ cannot stabilize unless the Alexander polynomial has degree 0 or 2 . The above speculation 
is also confirmed in $[\mathrm{C}]$.

We remark that if $n$-solvable is replaced by rationally $n$-solvable (see [COT, section 4]) then the analogous result holds without a condition on the Arf invariant of $J$. This is of interest in studying knots which bound disks in rational homology balls.

We now want to show that many of these families of knots are different, even up to concordance. For this purpose we consider the von Neumann signature invariants of section 2 [COT, section 5].

Suppose one is given homomorphisms $\phi: \pi_{1}(M) \rightarrow \Gamma, \phi_{J}: \pi_{1}\left(M_{J}\right) \rightarrow \Gamma$ such that $\phi([\eta])=\phi_{J}\left(\left[\mu_{J}\right]\right)$ and $\Gamma$ is a PTFA group. Then, if $M^{\prime}$ is as above, a unique $\phi^{\prime}: \pi_{1}\left(M^{\prime}\right) \rightarrow \Gamma$ is induced which extends $\phi$ and $\phi_{J}$.

Proposition 3.2. Given $K, J, \eta, \phi, \phi_{J}$ as above,

$$
\rho\left(M^{\prime}, \phi^{\prime}\right)=\rho(M, \phi)+\rho\left(M_{J}, \phi_{J}\right)
$$

whenever the right-hand side is defined.

Proof of Proposition 3.1. Let $W$ be an $n$-solution for $K$ and let $W_{J}$ be the 0 -solution for $J$ with $\pi_{1} \cong \mathbb{Z}$ discussed above Proposition 2.6. So $\partial W_{J}=M_{J}=E_{J} \cup\left(S^{1} \times D^{2}\right)$ where $S^{1} \times\{*\}$ is $\mu_{J}$ and $\{*\} \times \partial D^{2}$ is $\ell_{J}$. Let $W^{\prime}$ be the 4 -manifold obtained from $W_{J}$ and $W$ by identifying the solid torus $S^{1} \times D^{2} \subset \partial W_{J}$ with $\eta \times D^{2} \subset \partial W$. Observe that $\partial W^{\prime}=M^{\prime}$, the zero surgery on $K^{\prime}=K(J, \eta)$. We claim that $W^{\prime}$ is an $n$-solution for $K^{\prime}$. First consider the Mayer-Vietoris sequence below

$$
0 \longrightarrow H_{2}(W) \oplus H_{2}\left(W_{J}\right) \stackrel{\pi_{*}}{\longrightarrow} H_{2}\left(W^{\prime}\right) \stackrel{\partial_{*}}{\longrightarrow} H_{1}\left(W \cap W_{J}\right) \stackrel{i_{*}}{\longrightarrow} H_{1}(W) \oplus H_{1}\left(W_{J}\right)
$$

Since $W \cap W_{J} \simeq S^{1}, H_{2}\left(W \cap W_{J}\right)=0$ for any coefficients. Since the inclusion $W \cap W_{J} \rightarrow W_{J}$ induces an isomorphism on $\pi_{1}$, the map $i_{*}$ is a monomorphism with any coefficients. Thus $\pi_{*}$ is an isomorphism with any coefficients, and the intersection and self-intersection forms on $\mathrm{H}_{2}$ split naturally. We may think of an $n$-Lagrangian with its $n$-duals for $W$ as being generated by finite collections of based surfaces in $W$ each of which lifts to the $\pi_{1}(W)^{(n)}$-cover (these were called " $n$-surfaces" in [COT]; sections 7-8). These same surfaces are clearly $n$-surfaces in $W^{\prime}$ since $n^{\text {th }}$-order commutators in $\pi_{1}(W)$ are $n^{\text {th }}$-order commutators in $\pi_{1}\left(W^{\prime}\right)$. Similarly consider the collection of "0-surfaces" generating a 0-Lagrangian and its duals for $W_{J}$. Since the map $\pi_{1}\left(W_{J}\right) \rightarrow \pi_{1}\left(W^{\prime}\right) / \pi_{1}\left(W^{\prime}\right)^{(n)}$ is trivial (since $\pi_{1}\left(W_{J}\right)$ is generated by $\eta$ ), these 0 -surfaces are $n$-surfaces in $W^{\prime}$. It then follows easily by naturality that the union of these collections of $n$-surfaces constitutes an $n$-Lagrangian with $n$-duals for $W^{\prime}$ (see [COT]; sections 7-8).

Proof of Proposition 3.2. Suppose $\partial(W, \psi)=(M, \phi)$ and $\partial\left(W_{J}, \psi_{J}\right)=\left(M_{J}, \phi_{J}\right)$ for some $H_{1}$-bordisms as described in Section 2. Note that $M_{J}=E_{J} \cup\left(S^{1} \times\right.$ $D^{2}$ ) where $\{1\} \times \partial D^{2}$ is a longitude of $J$. Let $W^{\prime}$ be the 4 -manifold obtained from $W_{J}$ and $W$ by identifying the solid torus $S^{1} \times D^{2} \subset \partial W_{J}$ with $\eta \times D^{2} \subset \partial W$. Observe that $\partial W^{\prime}=M^{\prime}$ and that $\psi$ and $\psi_{J}$ piece together to give an extension 
of $\phi^{\prime}$ to $\psi^{\prime}: \pi_{1}\left(W^{\prime}\right) \rightarrow \Gamma$. Thus $\left(W^{\prime}, \psi^{\prime}\right)$ can be used to compute $\rho\left(M^{\prime}, \phi^{\prime}\right)$. It now suffices to show that the natural inclusions induce an isomorphism $H_{2}(W) \oplus$ $H_{2}\left(W_{J}\right) \rightarrow H_{2}\left(W^{\prime}\right)$ with $\mathcal{K}$ coefficients. As above, $H_{2}\left(W \cap W_{J}\right)=0$ with any coefficients. Now, appealing to the Mayer-Vietoris sequence, it will suffice to show that $H_{1}\left(W \cap W_{J} ; \mathcal{K}\right) \rightarrow H_{1}\left(W_{J} ; \mathcal{K}\right)$ is a monomorphism. If $\phi\left(\left[\mu_{J}\right]\right)=\phi([\eta]) \neq 0$ then $H_{1}\left(W \cap W_{J} ; \mathcal{K}\right)=0$ since the induced $\Gamma$-cover of a circle is a union of lines (alternatively use [COT]; Proposition 2.11). If $\phi\left(\left[\mu_{J}\right]\right)=0$ then $\phi_{J}$ is the zero map since $\pi_{1}\left(M_{J}\right)$ is normally generated by $\left[\mu_{J}\right]$. Hence we may assume $\psi_{J}$ is the zero homomorphism. Therefore the coefficient system $\psi_{J}: \mathbb{Q}\left[\pi_{1}\left(W_{J}\right)\right] \rightarrow \mathcal{K}$ factors as the augmentation $\epsilon: \mathbb{Q}\left[\pi_{1}\left(W_{J}\right)\right] \rightarrow \mathbb{Q}$ followed by the inclusion $\mathbb{Z}=$ $\mathbb{Q}[\{1\}] \hookrightarrow \mathbb{Q} \Gamma \hookrightarrow \mathcal{K}$. Thus $H_{1}\left(W_{J} ; \mathcal{K}\right) \cong H_{1}\left(W_{J} ; \mathbb{Q}\right) \otimes_{\mathbb{Q}} \mathcal{K} \cong \mathcal{K}$ and similarly for $H_{1}\left(W \cap W_{J} ; \mathcal{K}\right)$. Since the inclusion $W \cap W_{J} \rightarrow W_{J}$ induces an isomorphism on $H_{1}\left(\_; \mathbb{Q}\right)$, it induces an isomorphism on $H_{1}\left(\_; \mathcal{K}\right)$ as well.

The following application is what we will use in the proof of the main theorem.

Example 3.3. Suppose $K$ is a ribbon knot, $\eta \in \pi_{1}(M)^{(n)}$ and $J$ has Arf invariant zero. Then $K^{\prime}=K(J, \eta)$ is $n$-solvable by Proposition 3.1. Let $W$ be the exterior of a ribbon disk for $K$ and let $W_{J}$ be the (0)-solution for $J$ with $\pi_{1}\left(W_{J}\right) \cong \mathbb{Z}$ as in section 2. Then let $W^{\prime}$ be the $n$-solution for $K^{\prime}$ formed as in the proof of Proposition 3.1 by gluing $W_{J}$ to $W$ along $\eta \times D^{2}$. Suppose $\psi^{\prime}: \pi_{1}\left(W^{\prime}\right) \rightarrow \Gamma$ is a homomorphism defining, by restriction, $\phi^{\prime}, \psi, \phi_{J}$ and $\psi_{J}$ from (respectively) $\pi_{1}\left(M^{\prime}\right), \pi_{1}(W), \pi_{1}\left(M_{J}\right)$, and $\pi_{1}\left(W_{J}\right)$. Then $\rho\left(M^{\prime}, \phi^{\prime}\right)=\rho\left(M_{J}, \phi_{J}\right)$ by Proposition 3.2 and 2.2. Then, (since $\pi_{1}\left(W_{J}\right) \cong \mathbb{Z}$ is generated by $\eta$ ) using $2.3,2.4$, and $2.5, \rho\left(M^{\prime}, \phi^{\prime}\right)$ equals $\rho(J)$ if $\phi(\eta) \neq 1$ and equals 0 if $\phi(\eta)=1$.

\section{The Main Theorem}

Theorem 4.1. $\mathcal{F}_{(2)} / \mathcal{F}_{(2.5)}$ has infinite rank.

Proof. It is sufficient to exhibit an infinite set of (2.0)-solvable knots $K_{i}, i \in$ $\mathbb{Z}_{+}$, such that no non-trivial linear combination is (2.5)-solvable. Let $K_{r}$ be the "seedling" ribbon knot shown in Figure 3.a and $\eta$ be the designated circle just as was used in [COT, section 6]. Let $J_{i}$ be the knots of Proposition 2.6 and let $K_{i}=K_{r}\left(J_{i}, \eta\right)$ be the family of knots resulting from the grafting construction of Example 3.3. It was shown in [COT, section 6] that each of these knots is 2 -solvable and not (2.5)-solvable. Suppose that a non-trivial linear combination $\#_{i=1}^{m} n_{i}^{\prime} K_{i}, n_{i}^{\prime} \neq 0$, were (2.5)-solvable. We shall derive a contradiction.

We may assume all $n_{i}^{\prime}>0$ by replacing $K_{i}$ by $-K_{i}$ if $n_{i}^{\prime}<0$. We may also assume that if $m=1$ then $n_{1}^{\prime}>1$. For simplicity let $M_{i}$ denote $M_{K_{i}}$, and note that $-M_{i}=M_{-K_{i}}$ because $-K_{i}$ can be obtained by applying a reflection to $\left(S^{3}, K_{i}\right)$. Let $M_{0}$ denote the zero surgery on $\# n_{i}^{\prime} K_{i}$ and let $W_{0}$ denote the 
putative (2.5)-solution.

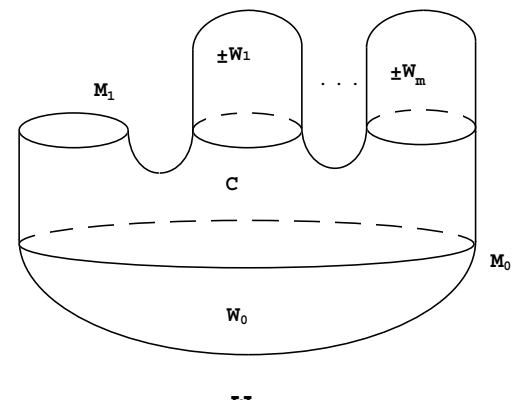

W

Figure 2

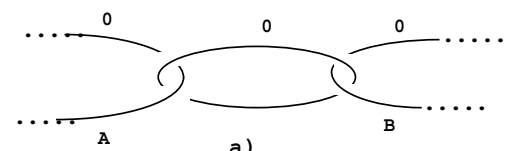

a)

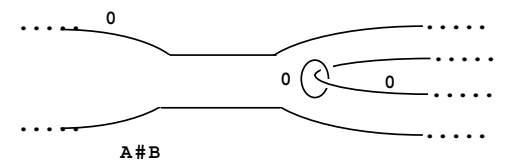

b)

Figure 3

From these assumptions we construct a very specific 2-solution $W$ for $M_{1}$. This is shown schematically in Figure 2 where $C$ and $W_{i}$ are to be defined. The $W_{i}$, $i>0$, are the specific 2-solutions for $M_{i}$ constructed as in Example 3.3. There are $n_{i}$ copies of $W_{i}$ where $n_{1}=n_{1}^{\prime}-1$ and $n_{i}=n_{i}^{\prime}$ if $i>1$. The 4-manifold $C$ is merely a standard cobordism between zero surgery on a connected sum of knots and the disjoint union of the zero surgeries on its summands. For the case of just two knots $A \# B$, the manifold $C$ is described as follows. Beginning with a collar on $M_{A} \amalg M_{B}$, add a 1-handle to get a connected 4-manifold whose "upper" boundary is given by surgery on the split link $A \amalg B \hookrightarrow S^{3}$, each with zero framing. Next add a zero framed 2-handle along a circle as shown in Figure 3a. This completes the description of $C$ in this simple case. We need only show that the 3-manifold in Figure 3a is homeomorphic to $M_{A \# B}$. This is accomplished most easily in the language of Kirby's calculus of framed links [Ki]. By "sliding" the $A$ circle over the $B$ circle, one arrives at the equivalent description shown in Figure 3b. But now the circle labelled $B$ may be cancelled by the small linking circle, leaving only the desired zero surgery on the connected sum. By iterating this idea, one sees that our $C$ has a handlebody decomposition, relative to $\coprod_{i=1}^{m} n_{i}^{\prime} M_{i}$, consisting of $\left(\sum_{i=1}^{m}\left|n_{i}\right|\right)$ 1-handles and the same number of 2-handles. The 1-handles have 
no effect on $H_{1}$ or $H_{2}$, while the 2-handles serve to equate all the meridional generators of $H_{1}$ (and thus do not affect $H_{2}$ ). Hence $H_{1}(C ; \mathbb{Z}) \cong \mathbb{Z}$ and the inclusion from any of its boundary components induces an isomorphism on $H_{1}$; and $H_{2}(C) \cong H_{2}\left(\amalg n_{i}^{\prime} M_{i}\right)$. Moreover, in $H_{2}(C)$, the generator of $H_{2}\left(M_{0}\right)$ is equal to the sum of generators of $H_{2}$ of the other components of $\partial C$.

We now verify that $W$ is an $H_{1}$-bordism for $M_{1}$, and identify $H_{2}(W)$. The inclusions induce isomorphisms $H_{1}\left(M_{i} ; \mathbb{Z}\right) \rightarrow H_{1}\left(W_{i} ; \mathbb{Z}\right)$. It then follows that $H_{1}\left(M_{1}\right) \rightarrow H_{1}(W) \cong \mathbb{Z}$ is an isomorphism. Now consider a Mayer-Vietoris sequence for $W=C \cup W^{*}$ where $W^{*}=\overline{C-W}$. We see that $H_{1}\left(C \cap W^{*}\right) \rightarrow H_{1}\left(W^{*}\right)$ is injective by the remarks above. Note that the boundary map $H_{3}\left(W_{i}, M_{i}\right) \rightarrow$ $H_{2}\left(M_{i}\right)$ is an isomorphism since it is dual to the inclusion $H^{1}\left(W_{i}\right) \longrightarrow H^{1}\left(M_{i}\right)$. Thus for $i \geq 0, H_{2}\left(M_{i}\right) \rightarrow H_{2}\left(W_{i}\right)$ is the zero map. Therefore $H_{2}\left(C \cap W^{*}\right) \rightarrow$ $H_{2}\left(W^{*}\right)$ is the zero map. By the last sentence of the previous paragraph, $H_{2}\left(C \cap W^{*}\right) \rightarrow H_{2}(C)$ is surjective. It follows that

$$
H_{2}(W) \cong H_{2}\left(W^{*}\right) \cong H_{2}\left(W_{0}\right) \bigoplus_{i=1}^{m} n_{i} H_{2}\left(W_{i}\right) .
$$

It is not difficult to see that $W$ is a spin bordism since each individual piece is spin with 2 spin structures and $\Omega_{3}^{\text {spin }}(\mathbb{Z}) \cong \mathbb{Z}_{2}$ is given by the Arf invariant.

To show that $W$ is a 2 -solution for $M_{1}$, we must exhibit a 2-Lagrangian with 2duals. But this is obtained merely by taking the "union" of the 2-Lagrangians and 2-duals for $W_{0}$ and each $\pm W_{i}$ which appears as part of $W$. (Recall that since $W_{0}$ is a (2.5)-solution it is also a 2-solution). More precisely suppose, for example, that $\left\{L_{1}, \ldots, L_{m}\right\},\left\{D_{1}, \ldots, D_{m}\right\}$ are 2-surfaces in $W_{0}$ which generate the 2-Lagrangian and its dual 2-Lagrangian for $W_{0}$. In particular these surfaces lift to $W_{0}^{(2)}$ and so the image of $\pi_{1}\left(L_{i}\right)$ in $\pi_{1}\left(W_{0}\right)$ is contained in $\pi_{1}\left(W_{0}\right)^{(2)}$. Thus this image is contained in $\pi_{1}(W)^{(2)}$ and so these surfaces lift to $W^{(2)}$. Similarly by functoriality of intersection with twisted coefficients, these surfaces have the required intersection properties when considering the intersection form on $W$ with $\mathbb{Z}\left[\pi_{1}(W) / \pi_{1}(W)^{(2)}\right]$ coefficients. An identical argument is used for each $W_{i}$, making it clear that the union of these 2-surfaces represents a 2-Lagrangian and 2-duals for $W$, completing the demonstration that $W$ is a 2 -solution for $M_{1}$.

Since $W$ is a 2 -solution for $M_{1}$, Theorem 4.6 of [COT] guarantees the existence of certain non-trivial homomorphisms $\phi_{2}: \pi_{1}\left(M_{1}\right) \rightarrow \Gamma_{2}^{U}$, for a certain universal solvable group $\Gamma_{2}^{U}$, which extend to $\pi_{1}(W)$. Moreover, if $W$ is fixed, such homomorphisms actually factor through a much smaller group (the image of $\pi_{1}(W)$, for example). This improvement was mentioned in Remark 4.7 of [COT] and was discussed in detail in section 6 of that paper for precisely the case at hand. We shall repeat some of that argument. Let $\Gamma_{0}=\mathbb{Z}$ and let $\phi_{0}: \pi_{1}\left(M_{1}\right) \rightarrow \Gamma_{0}$ be the canonical epimorphism which extends uniquely to an epimorphism $\psi_{0}: \pi_{1}(W) \rightarrow \Gamma_{0}$. Recall that the classical Alexander module $\mathcal{A}_{0}\left(M_{1}\right)=H_{1}\left(M_{1} ; \mathbb{Q}\left[t, t^{-1}\right]\right)$ is isomorphic to $\mathbb{Q}\left[t, t^{-1}\right] /(p(t))^{2}$ where $p(t)=t^{-1}-3+t$ (this computation was discussed, but left to the reader in section 6 of $[\mathrm{COT}])$. Let $\mathcal{A}_{0}(W)=H_{1}\left(W ; \mathbb{Q}\left[t, t^{-1}\right]\right)$. By 
[COT, Theorem 4.4], since $W$ is a 1-solution for $M_{1}$, the kernel of the inclusioninduced map $j_{*}: \mathcal{A}_{0}\left(M_{1}\right) \rightarrow \mathcal{A}_{0}(W)$ is self-annihilating with respect to the Blanchfield form $B \ell_{0}$. Since $\mathcal{A}_{0}\left(M_{1}\right)$ has a unique proper submodule $P_{0}$, the latter is in fact this kernel. Choose a non-zero $p_{0} \in P_{0}$, inducing $\phi_{1}: \pi_{1}\left(M_{1}\right) \rightarrow \Gamma_{1}^{U}$ by [COT, Theorem 3.5] (recall $\Gamma_{1}^{U}=\mathbb{Q}(t) / \mathbb{Q}\left[t, t^{-1}\right] \rtimes \Gamma_{0}$ ). By [COT, Theorem 3.6] $\phi_{1}$ extends to $\psi_{1}: \pi_{1}(W) \rightarrow \Gamma_{1}^{U}$. Using the argument of ([COT], see just before Proposition 6.1), we can replace $\Gamma_{1}^{U}$ by a subgroup $\Gamma_{1}$ which contains the image of $\phi_{1}$ and is isomorphic to $\mathbb{Q}\left[t, t^{-1}\right] /\left(p(t)^{m}\right) \rtimes \Gamma_{0}$ for some positive integer $m$; replace $\phi_{1}$ by restricting its image and replace $\psi_{1}$ by a new map extending this restriction. We re-label these new maps by $\phi_{1}$ and $\psi_{1}$. Continuing as in [COT], we choose a subring $\mathcal{R}_{1}$ of the field of fractions of $\mathbb{Z} \Gamma_{1}$ where $\mathcal{R}_{1}=\left(\mathbb{Q}\left[\left[\Gamma_{1}, \Gamma_{1}\right]\right]-\{0\}\right)^{-1} \mathbb{Q} \Gamma_{1}$ and then set $\mathcal{A}_{1}\left(M_{1}\right)=H_{1}\left(M_{1} ; \mathcal{R}_{1}\right), \mathcal{A}_{1}(W)=H_{1}\left(W ; \mathcal{R}_{1}\right)$ using the coefficient systems $\phi_{1}$ and $\psi_{1}$ respectively. Then, since $W$ is a 2 -solution, the kernel of $j_{*}: \mathcal{A}_{1}\left(M_{1}\right) \rightarrow \mathcal{A}_{1}(W)$ is self-annihilating with respect to the non-singular linking form $B \ell_{1}$ [COT, Theorem 4.4]. By Proposition 6.1 of [COT], $\mathcal{A}_{1}\left(M_{1}\right)$ has a unique self-annihilating submodule $P_{1}$ which is therefore the kernel of $j_{*}$. Choose a non-zero element $p_{1} \in P_{1}$ (by [COT, Proposition 6.2b] $P_{1}$ is non-trivial). This induces $\phi_{2}: \pi_{1}\left(M_{1}\right) \rightarrow \Gamma_{2}\left(\Gamma_{2}=\mathcal{K}_{1} / \mathcal{R}_{1} \rtimes \Gamma_{1}\right.$ where $\mathcal{K}_{1}$ is the quotient field of $\left.\mathcal{R}_{1}\right)$. We note that the loop $\eta$ is chosen so that $\phi_{2}(\eta) \neq e$ (see below Proposition 6.1 in $[\mathrm{COT}]$ ). Since $W$ is a 2 -solution for $M_{1}$, Theorem 3.6.1 of [COT] applies (with $\left.n=2, x=p_{1}\right)$ to show that $\phi_{2}$ extends to $\psi_{2}: \pi_{1}(W) \rightarrow \Gamma_{2}$. Therefore $\rho\left(M_{1}, \phi_{2}\right)$ is defined and can be computed using $\left(W, \psi_{2}\right)$.

We shall now compute $\rho\left(M_{1}, \phi_{2}\right)$ using $\left(W, \psi_{2}\right)$. Let $\phi_{(i, j)}$ denote the restriction of $\psi$ to the $j^{\text {th }}$ copy of $\pi_{1}\left(M_{i}\right) 1 \leq j \leq n_{i}$, and let $\phi_{0}$ denote the restriction of $\psi$ to $\pi_{1}\left(M_{0}\right)$. Note that each of these homomorphisms is non-trivial since the generator of $H_{1}(C ; \mathbb{Z}) \cong H_{1}\left(M_{1} ; \mathbb{Z}\right) \cong \mathbb{Z}$ is carried by each $M_{i}$ and since $\phi_{2}$ and $\psi_{2}$ agree on the abelianizations. Let $\mathcal{K}_{2}$ denote the quotient (skew) field of $\mathbb{Z} \Gamma_{2}$. Consequently $H_{*}\left(M_{i} ; \mathcal{K}_{2}\right)=0$ for $i \geq 0$ (Propositions 2.9 and 2.11 of [COT]), and hence a Mayer-Vietoris sequence shows that $H_{2}\left(W ; \mathcal{K}_{2}\right) \cong H_{2}\left(W_{0} ; \mathcal{K}_{2}\right) \oplus$ $H_{2}\left(C ; \mathcal{K}_{2}\right) \oplus H_{2}\left(W_{1} ; \mathcal{K}_{2}\right) \cdots \oplus H_{2}\left(W_{m} ; \mathcal{K}_{2}\right)$ where $-W_{i}$ occurs $n_{i}$ times and the coefficient systems on the subspaces of $W$ are induced by inclusion. Similarly the intersection form on $H_{2}\left(W ; \mathcal{K}_{2}\right)$ splits as such a direct sum. Moreover we claim that $H_{2}\left(C ; \mathcal{K}_{2}\right)=0$. Let $\partial_{+} C=\partial W_{0}$ and $\partial_{-} C=\coprod n_{i}^{\prime} M_{i}$. We have observed that $\left(C, \partial_{-} C\right)$ is a relative 2-complex with an equal number of 1-handles and 2-handles. The claim will follow from Lemma 4.2 which shows that, even though $C$ is not an integral homology cobordism, it is a $\mathcal{K}_{2}$-homology cobordism.

Lemma 4.2. Suppose $(C, \partial C)$ is a compact, oriented 4-dimensional Poincaré complex such that $\partial C=\partial_{+} C \amalg \partial_{-} C,\left(C, \partial_{-} C\right)$ is homotopy equivalent to a finite (relative) 2-complex with no 0-handles and a equal number of 1-and 2-handles. Suppose also that $\beta_{1}\left(\partial_{+} C\right)=1$ and that $\phi: \pi_{1}(C) \rightarrow \Gamma$ is non-trivial on $\pi_{1}\left(\partial_{-} C\right)$ where $\Gamma$ is a poly-torsion-free-abelian group with quotient field $\mathcal{K}_{\Gamma}$. Then

$$
H_{*}\left(C, \partial_{+} C ; \mathcal{K}_{\Gamma}\right) \cong H_{*}\left(C, \partial_{-} C ; \mathcal{K}_{\Gamma}\right) \cong H_{*}\left(C ; \mathcal{K}_{\Gamma}\right) \cong 0 .
$$


Proof. It follows that $\phi$ is non-trivial on $\pi_{1}(C)$ and $\pi_{1}\left(\partial_{+} C\right)$ and so $H_{0}(C ; \mathcal{K}) \cong$ $H_{0}\left(\partial_{+} C ; \mathcal{K}\right) \cong H_{0}\left(\partial_{-} C ; \mathcal{K}\right) \cong H_{0}\left(C, \partial_{+} C ; \mathcal{K}\right) \cong H_{0}\left(C, \partial_{-} C ; \mathcal{K}\right) \cong 0$ by $[\mathrm{COT}$, Proposition 2.9]. Moreover $H_{1}\left(C, \partial_{+} C ; \mathcal{K}\right) \cong H^{3}\left(C, \partial_{-} C ; \mathcal{K}\right) \cong 0$ since $\left(C, \partial_{-} C\right)$ is a 2-complex. Since $\beta_{1}\left(\partial_{+} C\right)=1, H_{1}\left(\partial_{+} C ; \mathcal{K}\right)=0$ by [COT, Proposition 2.11] and $H_{2}\left(\partial_{+} C ; \mathcal{K}\right) \cong H^{1}\left(\partial_{+} C ; \mathcal{K}\right) \cong 0$ by Remark 2.8 .3 of that paper. Combining this with the previous facts, we see that $H_{1}(C ; \mathcal{K}) \cong 0$ and hence that $H_{1}\left(C, \partial_{-} C ; \mathcal{K}\right) \cong$ 0 by consider the sequence of the pair $\left(C, \partial_{-} C\right)$. Finally, note that the chain complex obtained from the cell-structure of the 2-complex $\left(C, \partial_{-} C\right)$, by lifting to the $\Gamma$-cover and tensoring with $\mathcal{K}$, has only two non-zero terms, which are free $\mathcal{K}$-modules of the same rank. Since $H_{1}$ of this chain complex is zero, the boundary map $\partial_{2}$ is an epimorphism and hence an isomorphism. Thus $H_{2}\left(C, \partial_{-} C ; \mathcal{K}\right)=0$ which implies $H_{2}(C ; \mathcal{K})$ vanishes, implying $H_{2}\left(C, \partial_{+} C ; \mathcal{K}\right)=0$, and the claimed results in relative homology and hence homology now follow.

Thus, since $\sigma^{(2)}$ is a homomorphism on Witt classes of non-singular forms with $\mathcal{K}_{2}$ coefficients, we see that

$$
\rho\left(M_{1}, \phi_{2}\right)=\rho\left(M_{0}, \phi_{0}\right)+\sum_{i=1}^{m} \sum_{j=1}^{n_{i}} \rho\left(-M_{i}, \phi_{(i, j)}\right) .
$$

Since $\phi_{0}$ extends to the (2.5)-solution $W_{0}, \rho\left(M_{0}, \phi_{0}\right)$ vanishes by (2.2). Let $\epsilon_{i j}$ equal 0 or 1 according as $\phi_{(i, j)}(\eta)$ equals 0 or not. Since $\phi_{(i, j)}$ extends to $-W_{i}$, Example 3.3 establishes that $\rho\left(-M_{i}, \phi_{(i, j)}\right)=-\epsilon_{i j} \rho\left(J_{i}\right)$. This shows that $\rho\left(M_{1}, \phi_{2}\right)+\sum_{i=1}^{m} c_{i} \rho\left(J_{i}\right)=0$ where $c_{i}=\sum_{j=1}^{n_{i}} \epsilon_{i j}$ is non-negative.

It will now suffice to show that $\rho\left(M_{1}, \phi_{2}\right)$ equals $\rho\left(J_{1}\right)$, for this will, for any $c_{i}$, contradict Proposition 2.6. The argument of [COT, section 6] (outlined earlier in this proof as regards extension to $W$ ) shows that $\phi_{2}$ extends to the manifold $W_{1}$ as constructed in Example 3.3, the crucial facts being that $W_{1}$ is a 2-solution and the uniqueness of the self-annihilating submodules for the ordinary and firstorder generalized Alexander modules of $K_{1}$. Hence, by $2.3 \rho\left(M_{1}, \phi_{2}\right)=\rho\left(J_{1}\right)$ since $\phi_{2}(\eta) \neq e$. This contradiction establishes that no non-trivial linear combination of the $K_{i}$ is (2.5)-solvable and hence proves the Theorem.

\section{Appendix: Some results on slice knots}

The purpose of this section is to prove the following two results, as well as to prove Proposition 2.6. Only the latter is required for our main theorem.

Proposition 5.1. For $\omega \in S^{1}$, let $\sigma_{\omega}$ be the Levine-Tristram signature of a knot $K$. Then the (reduced) von Neumann signature of $K$ (denoted $\rho(K)$ in Section 2) is the integral of these signatures $\sigma_{\omega}$, integrated over the circle normalized to length one. 
Theorem 5.2. Suppose $K$ is a 1.5-solvable knot (for example a slice knot) whose Alexander polynomial is not 1 and which admits a Seifert surface $F$ of genus 1. Then there exists a homologically essential simple closed curve, $J$, on $F$ that has self-linking number zero and such that the integral of the Levine-Tristram signature function of $J$ vanishes.

The above theorem was announced in [COT]. In the case of slice knots, it first appeared in the unpublished Ph.D. thesis of D. Cooper and can also be compared to [Gi, Theorem 4]. It is not known if the latter theorem implies ours or vice-versa.

Proof of Theorem 5.2. Suppose $W$ is a 1.5-solution for $M$, the zero framed surgery on $K$. Let $G=\pi_{1}(W)$, let $\mathfrak{A}=G^{(1)} / G^{(2)} \otimes_{\mathbb{Z}\left[t, t^{-1}\right]} \mathbb{Q}\left[t, t^{-1}\right]$, and let $\Gamma=\mathfrak{A} \rtimes \mathbb{Z}$. Since $\mathfrak{A}$ is a torsion-free abelian group, $\Gamma$ is a PTFA (poly-torsion-free-abeliangroup) and is 1-solvable. Moreover there is a canonical homomorphism $\psi: G \rightarrow \Gamma$ since $G / G^{(2)} \cong\left(G^{(1)} / G^{(2)}\right) \rtimes \mathbb{Z}$. Let $\phi: \pi_{1}(M) \rightarrow \Gamma$ be the composition of $j_{*}: \pi_{1}(M) \rightarrow \pi_{1}(W)$ with $\psi$. By $2.2, \rho(M, \phi)=0$. Since $H_{1}(M) \cong H_{1}(W) \cong$ $H_{1}(\Gamma) \cong \mathbb{Z}$ we can consider the inclusion induced map on infinite cyclic covers, $j_{*}: H_{1}\left(M ; \mathbb{Q}\left[t, t^{-1}\right]\right) \rightarrow H_{1}\left(W ; \mathbb{Q}\left[t, t^{-1}\right]\right)$. The former group is isomorphic to the classical rational Alexander module $\mathcal{A}$ of $K$ (since the longitude lies in the second derived) and the latter is the group denoted $\mathfrak{A}$ above. Since $W$ is a 1 -solution for $M$, the kernel of $j_{*}$ is a submodule which is self-annihilating with respect to the classical Blanchfield form (apply [COT, Theorem 4.4] with $n=1$ and $\Gamma=\mathbb{Z}$ ). This fact is well known in case $W$ is the exterior of a slice disk for $K$. In particular, this implies that the kernel of $j_{*}$ and the image of $j_{*}$ have rank (over $\mathbb{Q}$ ) equal to one-half the degree of the Alexander polynomial, which is, by assumption, positive. Moreover this implies that $K$ is algebraically slice. The following algebraic fact about a genus 1 algebraically slice knot can be found in [Gi, Section 5]. $\mathcal{A}$ is a cyclic module with precisely 2 proper submodules each generated by a simple closed curve on $F$ of zero self-linking number. Let $J$ denote the proper submodule contained in kernel $\left(j_{*}\right)$. It follows that $J$ represents an element of kernel $\phi$. Now construct a cobordism over $\Gamma$ from $(M, \phi)$ to $\left(M_{J}, \phi^{\prime}\right)$ as follows, where $M_{J}$ is zero surery on $J$. To $M \times\{1\} \subset M \times[0,1]$ attach a zero-framed 2-handle along $J \times\{1\}$. The result of such a surgery can be seen to be homeomorphic to $M_{J} \# S^{1} \times S^{2}$ by sliding the zero-framed 2-handle $K \times\{1\}$ twice over $J$ so that it becomes unknotted and unlinked from $J$. Adding a 3-handle along $\{p t\} \times S^{2}$ completes the cobordism. Since $J \subset \operatorname{ker} \phi, \phi$ extends over the cobordism, inducing $\phi^{\prime}: \pi_{1}\left(M_{J}\right) \rightarrow \Gamma$. Since $\pi_{1}\left(M_{J}\right)$ is normally generated by the meridian of $J$, which clearly is null-homologous in the cobordism, $\phi^{\prime}$ factors through $[\Gamma, \Gamma]=\mathfrak{A}$. Since $H_{1}\left(M_{J}\right) \cong \mathbb{Z}$, the image of $\phi^{\prime}$ is either 0 or $\mathbb{Z}$. We claim it is the latter. For since the image of $j_{*}$ is not zero, there is an element of the Alexander module that maps non-trivially under $j_{*}$. Let $\gamma \in \pi_{1}(M-F)$ represent this element. Then $\gamma$ maps non-trivially under $\phi$. Since the 2 and 3-handles above are attached disjointly from $M-F, \gamma$ represents an element of $\pi_{1}\left(M_{J}\right)$ which maps non-trivially under 
$\phi^{\prime}$. Thus the image of $\phi^{\prime}$ is $Z$. By Lemma 4.2 , this cobordism is a $\mathcal{K}_{\Gamma}$-homology cobordism and thus $0=\rho(M, \phi)=\rho\left(M_{J}, \phi^{\prime}\right)$. Moreover $\rho\left(M_{J}, \phi^{\prime}\right)=\rho(J)$ which equals the integral of the signature function of $J$ (by 2.3, 2.4 and Proposition 5.1).

We now move to the proof of Proposition 5.1. Note that even though the $\sigma_{\omega}$ are integers, the integral is in general only a real number. We first give the relevant definitions and then derive Proposition 5.1 from Lemma 5.4.

First recall that the Levine-Tristram signature is defined as follows: Pick a Seifert surface $F$ for the knot, together with a basis of embedded curves $a_{1}, \ldots, a_{2 g}$ on $F$. Using the positive push-off's $a_{i}^{\uparrow}$ of $a_{i}$ into 3 -space, the corresponding Seifert matrix is defined using linking numbers in $S^{3}$ :

$$
S_{i j}:=l k\left(a_{i}, a_{j}^{\uparrow}\right) .
$$

Then for $\omega \in S^{1}, \sigma_{\omega}$ is the signature of the complex hermitian form

$$
\lambda_{\omega}(F):=\left(1-\omega^{-1}\right) \cdot S+(1-\omega) \cdot S^{T} .
$$

Instead of choosing a Seifert surface for the knot $K$, one can also choose a 4-manifold $W$ which bounds the 0 -surgery $M_{K}$ on $K$. One can also arrange that $W$ has signature zero (by adding copies of $\pm \mathbb{C P}^{2}$ ) and that $\pi_{1} W \cong \mathbb{Z}$, generated by a meridian $t$ of the knot. This follows from the fact that the bordism group $\Omega_{3}\left(S^{1}\right)$ vanishes.

For the purpose of this section, let's call a 4-manifold $W$ as above a $\mathbb{Z}$-bordism for the knot $K$. The following twisted signatures are associated to it: Define $\sigma_{\omega}(W)$ to be the signature of the intersection form $\lambda_{\omega}(W)$ on $H_{2}\left(W ; \mathbb{C}_{\omega}\right)$, where $\mathbb{C}_{\omega}$ is the module over $\mathbb{Z}\left[\pi_{1} W\right]=\mathbb{Z}\left[t, t^{-1}\right]$ obtained by letting $t$ act on $\mathbb{C}$ via multiplication by $\omega$. The isomorphism $\Omega_{4}\left(S^{1}\right) \cong \mathbb{Z}$, given by the (untwisted!) signature, implies by the additivity and bordism invariance of all twisted signatures that the signatures of $\lambda_{\omega}(W)$ are in fact independent of the $\mathbb{Z}$-bordism $W$ for $K$.

The following result was proven in [COT, Section 5] and will be used in order to avoid having to recall the definition of the von Neumann signature (or $L^{2}$ signature). Note however that the (reduced) $L^{2}$-signature of a knot is by definition the (reduced) $L^{2}$-signature of a $\mathbb{Z}$-bordism $W$. By [COT, Lemma 5.9.4] this is independent of the choice of $W$. Note also that since we have required that the ordinary signature of $W$ is zero, the reduced and unreduced $L^{2}$-signatures are identical.

Lemma 5.3. Let $W$ be a $\mathbb{Z}$-bordism for a knot $K$. Then the $L^{2}$-signature of $K$ is the integral of the twisted signatures $\sigma_{\omega}(W)$, integrated over the circle normalized to length one.

In order to connect to the setting of [COT, Section 5], note that the universal coefficient spectral sequence implies that for any $\mathbb{C}\left[t, t^{-1}\right]$-module $\mathcal{M}$ there is an 
isomorphism

$$
H_{2}(W ; M) \cong H_{2}\left(W ; \mathbb{C}\left[t, t^{-1}\right]\right) \otimes_{\mathbb{C}\left[t, t^{-1}\right]} \mathcal{M}
$$

First apply this isomorphism to $\mathcal{M}=\mathcal{U} \mathbb{Z}$, the algebra of unbounded operators affiliated to the von Neumann algebra $\mathcal{N} \mathbb{Z}$. It follows that the intersection form used in [COT, Def.5.8] to define the $L^{2}$-signature of $W$ is nothing else but $\lambda_{W} \otimes \mathcal{U} \mathbb{Z}$, where $\lambda_{W}$ is Wall's $\mathbb{Z}\left[t, t^{-1}\right]$-valued intersection form on $H_{2}\left(W ; \mathbb{Z}\left[t, t^{-1}\right]\right)$. This implies by [COT, Lemma 5.6] that the $L^{2}$-signature of $W$ is the $L^{2}$-signature of the form $\lambda_{W} \otimes_{\mathbb{Z}} \mathbb{C}$ on the free $\mathbb{C}\left[t, t^{-1}\right]$-module $H_{2}\left(W ; \mathbb{C}\left[t, t^{-1}\right]\right) /$ Torsion.

Finally, apply the isomorphism above to $\mathcal{M}=\mathbb{C}_{\omega}$ and observe that the intersection form $\lambda_{\omega}$ is obtained from the $\mathbb{C}\left[t, t^{-1}\right]$-valued intersection form $\lambda_{W} \otimes \mathbb{C}$ by substituting $\omega$ for $t$. Hence the discussion of [COT, Def.5.3] and in particular Lemma 5.4 apply to prove Lemma 5.3.

We return to the proof of Proposition 5.1 above. It now suffices to prove the following lemma.

Lemma 5.4. For a given Seifert surface $F$ for $K$, there exists a $\mathbb{Z}$-bordism $W_{F}$ such that the signatures of $\lambda_{\omega}(F)$ and $\lambda_{\omega}\left(W_{F}\right)$ agree for all $\omega \in S^{1}$.

Proof. First observe that the equality is true for the untwisted case $\omega=1$ since both signatures are zero. This holds for any $\mathbb{Z}$-bordism $W$. All other signatures can be calculated after inverting the element $(1-t)$ since for $\omega \neq 1$, the twisted homology is given as follows:

$$
H_{2}\left(W ; \mathbb{C}_{\omega}\right) \cong H_{2}\left(W ; \mathbb{C}\left[t, t^{-1}\right]\right) \otimes_{\mathbb{C}\left[t, t^{-1}\right]} \mathbb{C}_{\omega} \cong H_{2}(W ; \Lambda) \otimes_{\Lambda} \mathbb{C}_{\omega}
$$

where $\Lambda:=\mathbb{C}\left[t, t^{-1},(1-t)^{-1}\right]$. The second isomorphism uses the fact that $\Lambda$ is a flat module over the ring $\mathbb{C}\left[t, t^{-1}\right]$. Note that we are using $\omega \neq 1$ to make $\mathbb{C}$ a module over $\Lambda$ (again denoted by $\mathbb{C}_{\omega}$ ).

We finish our proof by showing that for a given Seifert surface $F$ for $K$, there is a certain choice of $W=W_{F}$ such that the intersection form on $H_{2}\left(W_{F} ; \Lambda\right)$ is represented by the matrix

$$
\lambda(F):=\left(1-t^{-1}\right) \cdot S+(1-t) \cdot S^{T}
$$

where $S$ is again the Seifert matrix for a basis of embedded curves $a_{1}, \ldots, a_{2 g}$ on $F$. The first part of our computation follows [Ko] closely.

Let $V_{F}$ be the complement of a neighborhood of the Seifert surface $F$ pushed into the 4-ball $D^{4}$. We will later modify $V_{F}$ to get the desired 4-manifold $W_{F}$. $\pi_{1}\left(V_{F}\right)=\langle t\rangle \cong \mathbb{Z}$. We construct the universal cover of $V_{F}$ in the usual manner. Cut along the trace of pushing the surface $F$ into the 4 -ball. The cut manifold is homeomorphic to $D^{4}$. This determines two embeddings of $F \times I$ in the boundary of $D^{4}$ and we label the positive side $F_{+}$and the negative side $F_{-}$. Construct the universal cover of $V_{F}$ from countably many copies of $D^{4}$ labelled $t^{k} D^{4}, k \in \mathbb{Z}$ by identifying $F_{+} \subset t^{k} D^{4}$ with $F_{-} \subset t^{k-1} D^{4}$ in the obvious manner. Each 4-ball is a fundamental domain of this cover. 
The Mayer-Vietoris sequence of this decomposition gives an isomorphism of $\mathbb{Z}[\mathbb{Z}]$-modules

$$
H_{2}\left(V_{F} ; \mathbb{Z}[\mathbb{Z}]\right) \stackrel{\partial}{\rightarrow} H_{1}(F ; \mathbb{Z}) \otimes_{\mathbb{Z}} \mathbb{Z}[\mathbb{Z}] .
$$

Thus, $H_{2}\left(V_{F} ; \mathbb{Z}[\mathbb{Z}]\right)$ is a free $\mathbb{Z}[\mathbb{Z}]$ module generated by $H_{1}(F)$. Furthermore, a basis of $H_{2}\left(V_{F} ; \mathbb{Z}[\mathbb{Z}]\right) \cong \pi_{2}\left(V_{F}\right)$ is obtained from a basis of curves of $H_{1}(F ; Z)$, $a_{i} \subset F$, by choosing immersed 2-disks

$$
\left(\Sigma_{i}^{+}, \partial \Sigma_{i}^{+}\right) \subset\left(t D^{4}, F_{+}\right) \text {and }\left(\Sigma_{i}^{-}, \partial \Sigma_{i}^{-}\right) \subset\left(t^{0} D^{4}, F_{-}\right)
$$

and orienting $\Sigma_{i}:=\Sigma_{i}^{+} \cup \Sigma_{i}^{-}($and $F)$ so that

$$
\partial \Sigma_{i}^{+}=a_{i}^{\uparrow} \text { and } \partial \Sigma_{i}^{-}=-a_{i}^{\downarrow} .
$$

By the Mayer-Vietoris argument given above and the usual geometric interpretation of the connecting homomorphism, the spheres $\Sigma_{1}, \ldots, \Sigma_{2 g}$ together give a basis for the free $\mathbb{Z}[\mathbb{Z}]$ module $H_{2}\left(V_{F} ; \mathbb{Z}[\mathbb{Z}]\right)$. We now compute the equivariant intersection form $\lambda_{V_{F}}$ on $H_{2}\left(V_{F} ; \mathbb{Z}[\mathbb{Z}]\right) \cong \pi_{2}\left(V_{F}\right)$ given by

$$
\lambda_{V_{F}}\left(\Sigma_{i}, \Sigma_{j}\right)=\sum_{k}\left(\Sigma_{i} \cdot t^{k} \Sigma_{j}\right) t^{k} \in \mathbb{Z}[\mathbb{Z}],
$$

where $\cdot$ is the usual (integer valued) intersection number of the 2-spheres $\Sigma_{i}$ in the universal cover of $V_{F}$.

By general position, we may assume that $\Sigma_{i}$ and $\Sigma_{j}$ do not intersect each other along $F \times I$ (since they intersect $F \times I$ only in their boundary circles). Moreover, $\Sigma_{i} \cdot t^{k} \Sigma_{j}=0$ for $k \neq-1,0,1$. Recalling that $S_{i, j}=l k\left(a_{i}, a_{j}^{\uparrow}\right)$ is the $(i, j)$ entry of the Seifert matrix $S$ associated to the Seifert surface $F$, we now compute equivariant intersection numbers as follows.

$$
\begin{aligned}
\Sigma_{i} \cdot \Sigma_{j} & =\Sigma_{i}^{-} \cdot \Sigma_{j}^{-}+\Sigma_{i}^{+} \cdot \Sigma_{j}^{+}=l k\left(-a_{i}^{\downarrow},-a_{j}^{\downarrow}\right)+l k\left(a_{i}^{\uparrow}, a_{j}^{\uparrow}\right) \\
& =l k\left(a_{i}, a_{j}^{\downarrow}\right)+l k\left(a_{i}, a_{j}^{\uparrow}\right)=S_{j, i}+S_{i, j} .
\end{aligned}
$$

Note that by symmetry, this is independent of how the curves $a_{i}$ respectively $a_{j}$ are pushed into general position. Moreover, one has

$$
\begin{gathered}
\Sigma_{i} \cdot t \Sigma_{j}=\Sigma_{i}^{+} \cdot t \Sigma_{j}^{-}=l k\left(a_{i}^{\uparrow},-a_{j}^{\downarrow}\right)=-l k\left(a_{j}, a_{i}^{\uparrow}\right)=-S_{j, i}, \\
\Sigma_{i} \cdot t^{-1} \Sigma_{j}=\Sigma_{i}^{-} \cdot t^{-1} \Sigma_{j}^{+}=l k\left(-a_{i}^{\downarrow}, a_{j}^{\uparrow}\right)=-l k\left(a_{i}, a_{j}^{\uparrow}\right)=-S_{i, j} .
\end{gathered}
$$

Thus the intersection form on $H_{2}\left(V_{F} ; \mathbb{Z}[\mathbb{Z}]\right)$ is given by the matrix

$$
\lambda_{V_{F}}\left(\Sigma_{i}, \Sigma_{j}\right)=\left(1-t^{-1}\right) S_{i, j}+(1-t) S_{i, j}^{T}=\lambda(F)_{i, j}
$$

as claimed.

Even though it might look now like we are done, the 4-manifold $V_{F}$ isn't quite what we want since it's boundary is not $M_{K}$. By construction, $\partial V_{F}$ is obtained from the knot complement by attaching $F \times S^{1}$ (instead of $D^{2} \times S^{1}$ to get $M_{K}$.) We turn $V_{F}$ into a 4-manifold $W_{F}$ with the correct boundary by attaching round handles $\left(D^{2} \times D^{1}\right) \times S^{1}$ to a disjointly embedded half basis of curves on $F$. In 
other words, we start with $g$ surgeries turning $F$ into a 2-disk and then cross with the circle. Clearly the boundary of $W_{F}$ is $M_{K}$. What happened to the equivariant intersection form?

Here we make use of the ring $\Lambda$ to simplify life tremendously. Lemma 5.6 below and an easy Mayer-Vietoris sequence argument imply that the inclusion induces an isomorphism $H_{2}\left(V_{F} ; \Lambda\right) \cong H_{2}\left(W_{F} ; \Lambda\right)$, and thus concludes the proof of Proposition 5.1.

Remark 5.5. The above proof inverts $1-t$ to save a lot of work. The more geometric reader will find the intersection pairing for $W_{F}$ before localization computed in $[\mathrm{Ko}]$.

One should compare that work with the simplicity of the following

Lemma 5.6. Let $X$ be a finite $C W$-complex and $t$ be a generator of $\pi_{1} S^{1}$. If $\Lambda:=\mathbb{Z}\left[t, t^{-1},(1-t)^{-1}\right]$, then

$$
H_{*}\left(X \times S^{1} ; \Lambda\right)=0
$$

if the twisting is given by the projection $p_{2}: X \times S^{1} \rightarrow S^{1}$.

Proof. The differential in the cellular chain complex of the universal cover of $S^{1}=$ $e^{0} \cup e^{1}$ is given by multiplication with $(t-1)$. This is clearly an isomorphism after tensoring with $\Lambda$. Hence, this chain complex is acyclic, and thus contractible.

The cellular chain complex of the $\mathbb{Z}$-cover of $X \times S^{1}$ with $\Lambda$ coefficients is the tensor product of the cellular chains of $X$ with the contractible $\Lambda$-module chain complex for $S^{1}$. A tensor product with a contractible chain complex is again contractible, and therefor acyclic.

Remark 5.7. It is clear that the manifold $V_{F}$ constructed in Lemma 5.4 has a spin structure as a subset of the 4-ball. The reader is invited to check that the $\mathbb{Z}$-bordism $W_{F}$ is a spin manifold if and only if the original knot $K$ has trivial Arf invariant. For example, if the Arf invariant is trivial then one may choose a half basis of curves $a_{1}, \ldots, a_{g}$ on $F$ with even self-linking number. This implies that the round surgeries leading from $V_{F}$ to $W_{F}$ are spin structure preserving.

We now return to prove the technical Proposition 2.6.

Proof of 2.6. Let $J_{m}, m \geq 1$, be a knot with Alexander polynomial $\Delta(t)=2 m t-$ $(4 m-1)+2 m t^{-1}$. Such a knot necessarily has zero Arf invariant (see Theorem 10.4 of [Ka]). Let $M$ be the zero surgery on $J_{m}$ and $W$ be a compact, spin 4-manifold with $\partial W=M, \pi_{1}(W) \cong \mathbb{Z}$ and $j_{*}: H_{1}(M ; \mathbb{Z}) \longrightarrow H_{1}(W, \mathbb{Z})$ an isomorphism. By considering the long exact homology sequence for the pair $(W, M)$ with $\mathbb{Z}\left[t, t^{-1}\right]$ coefficients (using the canonical epimorphisms $\phi_{i}, \psi_{i}$ to $\mathbb{Z}$ to define the coefficient systems) one sees that the order of the Alexander module $H_{1}\left(M ; \mathbb{Q}\left[t, t^{-1}\right]\right)$ is the 
determinant of a matrix $h$ representing the intersection form on $H_{2}\left(W ; \mathbb{Q}\left[t, t^{-1}\right]\right)$. The (reduced) signature function $\sigma(h(z))-\sigma_{0}(h): S^{1} \longrightarrow \mathbb{Z}$ is a locally-constant function which is 0 at $z=1$ and changes value only (possibly) at the two zeros of $\operatorname{det} h(z)= \pm \Delta(z)$ which are $e^{ \pm i \theta_{m}}$, where $0 \leq \theta_{m} \leq \frac{\pi}{2}$ and $\cos \theta_{m}=(4 m-1) / 4 m$. Since $\operatorname{det} h(z)$ changes sign at $e^{i \theta_{m}}, \sigma(h(z))$ must change value there. Thus $\sigma(h(-1))=a_{m}$, for some non-zero integer $a_{m}$ (actually \pm 2 ), and $\rho\left(M_{m}, \phi_{m}\right)=$ $a_{m}\left(\pi-\theta_{m}\right) / \pi$. To prove 2.6 , it will suffice to prove that there exists a infinite collection of integers $m$ such that $\left\{\theta_{m}\right\}$ is linearly independent over the integers.

Choose primes $p_{i}, 5 \leq p_{1}<p_{2}<\cdots<p_{j}<\ldots$, each congruent to 1 modulo 4 , and set $m_{j}=\frac{1}{8}\left(p_{j}-1\right)^{2}$. We claim that if $\Theta_{j}=\cos ^{-1}\left(\frac{4 m_{j}-1}{4 m_{j}}\right)$, then $\left\{\Theta_{j}\right\}$ is linearly independent over the rationals.

Lemma 5.8. Suppose $5 \leq p_{1}<p_{2}<\cdots<p_{n}$ are primes. Let $\xi_{j}=i \sqrt{p_{j}\left(p_{j}-2\right)}$ where $i=\sqrt{-1}$. Then $\left[\overline{\mathbb{Q}}\left(\xi_{1}, \ldots, \xi_{n}\right): \mathbb{Q}\right]=2^{n}$ and the Galois group over $\mathbb{Q}$ is $\left(\mathbb{Z}_{2}\right)^{n}$ generated by automorphisms $\phi_{j}$ where $\phi_{j}\left(\xi_{\ell}\right)=\left\{\begin{array}{ll}-\xi_{\ell} & \text { if } \ell=j \\ \xi_{\ell} & \text { if } \ell \neq j\end{array}\right.$.

Proof of Lemma 5.8. One verifies easily that the Lemma is true for $n=1$. Now assume $\left[\mathbb{Q}\left(\xi_{1}, \ldots, \xi_{k-1}\right): \mathbb{Q}\right]=2^{k-1}$ for any increasing sequence of primes $5<$ $p_{1}<\cdots<p_{k-1}$. We shall show $\left[\mathbb{Q}\left(\xi_{1}, \ldots, \xi_{k}\right): \mathbb{Q}\right]=2^{k}$. It suffices to show $\xi_{k}$ is not in $\mathbb{Q}\left(\xi_{1}, \ldots, \xi_{k-1}\right)$. If it were then there are $\alpha, \beta \in \mathbb{Q}\left(\xi_{1}, \ldots, \xi_{k-2}\right)$ with $\xi_{k}=\alpha+\beta \xi_{k-1}$. Squaring each side yields

$$
\xi_{k}^{2}=\left(\alpha^{2}+\beta^{2} \xi_{k-1}^{2}\right)+2 \alpha \beta \xi_{k-1} .
$$

Since $\xi_{k}^{2}, \xi_{k-1}^{2}, \alpha$ and $\beta$ lie in $\mathbb{Q}\left(\xi_{1}, \ldots, \xi_{k-2}\right)$ and since, by the inductive hypothesis, $\xi_{k-1} \notin \mathbb{Q}\left(\xi_{1}, \ldots, \xi_{k-2}\right)$, we have $\alpha \beta=0$. If $\beta=0$ then $\xi_{k} \in \mathbb{Q}\left(\xi_{1}, \ldots, \xi_{k-2}\right)$. This will contradict the induction hypothesis for the sequence $5<p_{1}<\cdots<p_{k-2}<p_{k}$. Suppose $\alpha=0$. Since $\beta=\gamma+\delta \xi_{k-2}$ where $\gamma, \delta \in \mathbb{Q}\left(\xi_{1}, \ldots, \xi_{k-3}\right)$, by squaring each side of $\xi_{k}=\beta \xi_{k-1}$ and applying induction we again conclude $\gamma \delta=0$. As above if $\delta=0$ then $\beta \in \mathbb{Q}\left(\xi_{1}, \ldots, \xi_{k-3}\right)$ so $\xi_{k} \in \mathbb{Q}\left(\xi_{1}, \ldots, \xi_{k-3}, \xi_{k-1}\right)$ contradicting the induction hypothesis. Thus $\gamma=0$ and $\xi_{k}=\delta \xi_{k-2} \xi_{k-1}$. We continue in this fashion until we conclude $\xi_{k}=\Theta \xi_{1} \xi_{2} \ldots \xi_{k-1}$ where $\Theta \in \mathbb{Q}$, implying

$$
-p_{k}\left(p_{k}-2\right)=\left(r^{2} / s^{2}\right) \xi_{1}^{2} \ldots \xi_{k-1}^{2}
$$

Multiplying by $s^{2}$ yields an equation over $\mathbb{Z}$ which has no solution since $p_{k}$ divides the left-hand side an odd number of times and the right-hand side an even number of times. This contradiction finishes the proof that $\left[\mathbb{Q}\left(\xi_{1}, \ldots, \xi_{k}\right): \mathbb{Q}\right]=2^{k}$.

Consider the subgroup of the Galois group generated by the $\phi_{j}$. The subset $\left\{\phi_{i_{1}} \phi_{i_{2}} \ldots \phi_{i_{m}} \mid 1 \leq \phi_{i_{1}}<\cdots<\phi_{i_{m}} \leq n\right\}$ is easily seen to contain $2^{n}$ distinct elements of order 2 and the result follows.

Now suppose $\sum_{j=1}^{n} c_{j} \Theta_{j}=0$ where $c_{j} \in \mathbb{Z}$. Multiplying by $i$ and exponentiat- 
ing yields

$$
\prod_{j=1}^{n}\left(\frac{4 m_{j}-1}{4 m_{j}}+\frac{\xi_{j}}{4 m_{j}}\right)^{c_{j}}=1 .
$$

Evaluating $\phi_{j}$ on each side yields that $\left(\frac{4 m_{j}-1}{4 m_{j}}+\frac{\xi_{j}}{4 m_{j}}\right)^{c_{j}}$ is real and hence (being of norm 1) is \pm 1 . Thus $\frac{4 m_{j}-1}{4 m_{j}}+\frac{\xi_{j}}{4 m_{j}}$ is a primitive $r_{j}^{\text {th }}$ root of unity for some $r_{j} \mid c_{j}$. Since $\left[\mathbb{Q}\left(\xi_{j}\right), \mathbb{Q}\right]=2, r_{j}$ equals 3,4 or 6 . But, given the bound on $m_{j}$, one checks by inspection that this is not the case. This is a contradiction.

\section{References}

[CG] A. J. Casson and C. McA. Gordon, On slice knots in dimension three, Proc. Symp. in Pure Math. XXX, part 2 (1978) 39-53.

[COT] T. Cochran, K. E. Orr and P. Teichner, Knot concordance, Whitney towers and $L^{2}$ signatures, Annals of Math. 157 (2003), 433-519.

[C] T. Cochran, Noncommutative Knot Theory, preprint, Rice University, 2001, xxx math.GT/0206258.

[ChG] J. Cheeger and M. Gromov, Bounds on the von Neumann dimension of $L^{2}$-cohomology and the Gauss-Bonnet Theorem for open manifolds, J. Diff. Geometry 21 (1985), 1-34.

[CO $]$ T. Cochran and K. E. Orr, Homology boundary links and Blanchfield forms: Concordance Classification and new tangle-theoretic constructions, Topology 33 no. 3 (1994), 397-427.

[CT] T. Cochran and P. Teichner, Knot concordance and von-Neumann eta invariants, in preparation.

[Gi] P. Gilmer, Classical knot and link concordance, Comm. Math. Helv. 68 (1993), 1-19.

$[\mathrm{J}] \quad$ B. J. Jiang, A simple proof that the concordance group of algebraically slice knots is infinitely generated, Proc. Amer. Math. Soc. 83 no. 1 (1981), 189-192.

[Ka] L. Kauffman, On Knots, Annals of Math. Studies \#115, Princeton University Press, Princeton, N.J., 1987.

[Ko] K. H. Ko, A Seifert matrix interpretation of Cappell and Shaneson's approach to link cobordisms, Math. Proc. Cambridge Philos. Soc. 106 no. 3, (1986), 531-545.

[Ki] R. Kirby, The calculus of framed links in $S^{3}$, Inventiones Math. 45 (1978), 35-56.

[L] J. P. Levine, Knot cobordism groups in codimension two, Comm. Math. Helv. 44 (1969), 229-244.

[Li] C. Livingston, Seifert forms and Concordance, Geometric Topology 6 (2002), 403-408.

[Lt] R. Litherland, Cobordism of Satellite Knots, in: Four-Manifold Theory, Contemporary Mathematics no. 35 (C. Gordon and R. Kirby eds.), AMS, Providence, R.I., 1984.

[Wa] C. T. C. Wall, Surgery on Compact Manifolds, London Math. Soc. Monographs 1, Academic Press 1970.

Tim D. Cochran

Department of Mathematics

Rice University

P.O. Box 1892

Houston TX 77005-1892

USA

e-mail: cochran@rice.edu
Kent E. Orr

Department of Mathematics

Indiana University

47401 Bloomington, IN

USA

e-mail: korr@indiana.edu
Peter Teichner

Department of Mathematics

UC San Diego

9500 Gilmon Drive

La Jolla CA 92093-0112

USA

(Received: July 5, 2002) 